\title{
A remark on the minimal dispersion
}

\author{
A. E. Litvak
}

\begin{abstract}
We improve known upper bounds for the minimal dispersion of a point set in the unit cube and its inverse in both the periodic and non-periodic settings. Some of our bounds are sharp up to logarithmic factors.
\end{abstract}

AMS 2010 Classification: primary: 52B55, 52A23; secondary: 68Q25, 65Y20. Keywords: complexity, dispersion, largest empty box, torus

\section{Introduction and main results}

In this note we deal with the minimal dispersion of a point set in the unit cube. The dispersion of a point set in the $d$-dimensional unit cube $[0,1]^{d}$ is defined as the maximal volume of an axis parallel box in the cube which does not contain any point from the set. Then the minimal dispersion is a function of two variables, $n$ and $d$, which minimizes the dispersion over all possible choices of $n$ points. Such definition was introduced in [9] modifying a notion from [7]. Due to important applications and due to the fact that the problem is very interesting by itself, it has attracted a considerable attention in recent years. We refer to [1, 3, 5, 10, 11, 13, 15, 16] and references therein for the history of the problem and its relation to other branches as well as for the best known bounds (see also [8, 12, 14] for the dispersion of certain sets). We improve known upper bounds for the minimal dispersion and its inverse function. We will also consider the minimal dispersion on the torus and discuss the sharpness of our results. We would like to emphasize that we look at the dispersion as at a function of two variables, without trying to fix one of the variables. Instead, we consider both variables growing to infinity and our bounds depend on the relations between variables. The main novelty in our proof is a better construction of a family of axis parallel boxes (periodic or nonperiodic) needed to be checked for a random choice of points. It seems that our 
construction also leads to better bounds for recently introduced in [6] $k$-dispersion (where, given set of $n$ points, one allows axis parallel boxes to have inside at most $k$ points from this set), but we do not pursue this direction.

\section{$1.1 \quad$ Notations}

We start with notations. Given a measurable set $A \subset \mathbb{R}^{d}$, we denote its $d$ dimensional volume by $|A|$. We also use the same notation $|M|$ for the cardinality of a finite set $M$ (it always will be clear from the context what $|\cdot|$ means). By $\mathcal{R}_{d}$ we denote the set of all axis parallel boxes contained in the cube $Q_{d}:=[0,1]^{d}$, that is

$$
\mathcal{R}_{d}:=\left\{\prod_{i=1}^{d} I_{i} \mid I_{i}=\left[a_{i}, b_{i}\right) \subset[0,1]\right\} .
$$

The dispersion of a finite set $P \subset Q_{d}$ is defined as

$$
\operatorname{disp}(P)=\sup \left\{|B| \mid B \in \mathcal{R}_{d}, B \cap P=\emptyset\right\} .
$$

Then the minimal dispersion is defined as the function of two variables - the cardinality of a set of points $P \subset Q_{d}$ and the dimension, namely

$$
\operatorname{disp}^{*}(n, d)=\inf _{|P|=n} \operatorname{disp}(P)
$$

We also define its inverse as

$$
N(\varepsilon, d)=\min \left\{n \in \mathbb{N} \mid \operatorname{disp}^{*}(n, d) \leq \varepsilon\right\} .
$$

Since in our proofs we use a random choice of points, it will be natural to prove results in terms of the function $N(\varepsilon, d)$ and then to provide the corresponding (equivalent) bounds for the minimal dispersion itself.

\subsection{Known results.}

First we discuss the known bounds. In [1] it was shown that for $\varepsilon<1 / 4$,

$$
(1-4 \varepsilon) \frac{\log _{2} d}{4 \varepsilon} \leq N(\varepsilon, d) \leq \frac{2^{7 d+1}}{\varepsilon},
$$

where the upper bound is due to Larcher, improving the previous bound via primorials due to Rote and Tichy [9] (see also [3]) and the lower bound is the first non-trivial bound showing that the minimal dispersion grows with the dimension. Note that one trivially has $\operatorname{disp}^{*}(n, d) \geq 1 /(n+1)$, hence $N(\varepsilon, d) \geq 1 / \varepsilon-1$. 
Although estimates in (1) are tight when the dimension $d$ is small and $\varepsilon$ goes to 0 , there is a huge gap between the upper and lower bounds when the dimension starts to grow to infinity. Using random choice of points uniformly distributed in $Q_{d}$, Rudolf [10] obtained

$$
N(\varepsilon, d) \leq \frac{8 d}{\varepsilon} \log _{2}\left(\frac{33}{\varepsilon}\right)
$$

(this bound with different numerical constants also follows from much more general results in [2], where the $\mathrm{VC}$ dimension of $\mathcal{R}_{d}$ was used, and from the fact that this $\mathrm{VC}$ dimension equals to $2 d$ ). Estimate (2) is better than the upper bound in (1) in the regime

$$
\varepsilon \geq \exp \left(-C^{d}\right)
$$

where $C>1$ is an absolute constant (in this note we do not try to compute actual numerical values of absolute constants, that is, constants independent of any other parameters, one can find them following the proofs). Thus, if $\varepsilon$ is not extremely small with respect to the dimension, the gap in bounds is polynomial in $d$ and logarithmical in $1 / \varepsilon$. Another important feature of the Rudolf proof is that a random choice of points uniformly distributed on $Q_{d}$ gives the result.

It was natural to conjecture that $N(\varepsilon, d)$ behaves as $d / \varepsilon$, especially in view of corresponding bounds in the periodic setting (see below), however, surprisingly, Sosnovec [11] was able to improve the upper bound for $\varepsilon<1 / 4$ to

$$
N(\varepsilon, d) \leq C_{\varepsilon} \log _{2} d,
$$

where the order of magnitude of $C_{\varepsilon}$ was essentially $(1 / \varepsilon)^{(1 / \varepsilon)^{2}}$. This dependence was significantly improved in [15] by Ullrich and Vybíral, who showed that

$$
C_{\varepsilon}=\frac{2^{7}}{\varepsilon^{2}}\left(\log _{2}\left(\frac{1}{\varepsilon}\right)\right)^{2}
$$

works. They also conjectured that $N(\varepsilon, d)$ behaves as $\log d / \varepsilon$. The SosnovecUllrich-Vybíral upper bound is better in the regime

$$
\varepsilon \geq \frac{C\left(\log _{2} d\right)^{2}}{d}
$$

The Sosnovec-Ullrich-Vybíral proof is also based on a random choice of points, but instead of the uniform distribution on $Q_{d}$ they use uniform distribution on a certain lattice, gaining in the case of large $\varepsilon$. We discuss this in more details below. Let us also mention that in the same paper Sosnovec proved that the 
function $N(\varepsilon, d)$ completely changes the behaviour at $\varepsilon=1 / 4$, more precisely, he proved that for every $\varepsilon>1 / 4$,

$$
N(\varepsilon, d) \leq 1+\left\lfloor\frac{1}{\varepsilon-1 / 4}\right\rfloor
$$

Thus, for $\varepsilon>1 / 4$, the function $N(\varepsilon, d)$ is not growing with $d$. Note that clearly $N(1 / 2, d)=1$ (by taking the point $(1 / 2,1 / 2, \ldots, 1 / 2)$ ). One can summarize the previously known upper bounds for $\varepsilon \leq 1 / 4$ in

$$
N(\varepsilon, d) \leq \begin{cases}\frac{C \ln d}{\varepsilon^{2}} \ln ^{2}\left(\frac{1}{\varepsilon}\right), & \text { if } \varepsilon \geq \frac{\ln ^{2} d}{d}, \\ \frac{C^{d}}{\varepsilon} \ln \left(\frac{1}{\varepsilon}\right), & \text { if } \frac{\ln ^{2} d}{d} \geq \varepsilon \geq \exp \left(-C^{d}\right) \\ \frac{C^{d}}{\varepsilon}, & \text { if } \varepsilon \leq \exp \left(-C^{d}\right),\end{cases}
$$

where $1<C<1000$ is an absolute constant.

\subsection{New results}

In this note we improve the known bounds in the regime $\varepsilon \geq \exp \left(-C^{d}\right)$. Our first result improves bounds when $\varepsilon$ is not large.

Theorem 1.1. There exists an absolute constant $C \geq 1$ such that the following holds. Let $d \geq 2$ and $\varepsilon \in(0,1 / 2]$. Then

$$
\begin{array}{ll}
N(\varepsilon, d) \leq \frac{C \ln d}{\varepsilon} \ln \left(\frac{1}{\varepsilon}\right), & \text { provided that } \quad \varepsilon \leq \exp (-d), \\
N(\varepsilon, d) \leq \frac{C d}{\varepsilon} \ln \ln \left(\frac{2}{\varepsilon}\right), & \text { provided that } \quad \varepsilon \geq \exp (-d) .
\end{array}
$$

Moreover, the random choice of points with respect to the uniform distribution on the cube $Q_{d}$ gives the result with high probability.

We would like to emphasize that if $\varepsilon \leq \exp (-d)$ then, in view of (1), Theorem 1.1 yields

$$
\frac{\ln d}{6 \varepsilon} \leq N(\varepsilon, d) \leq \frac{C \ln d}{\varepsilon} \ln \left(\frac{1}{\varepsilon}\right),
$$

thus the gap in bounds is only logarithmical in $1 / \varepsilon$. In the second case the improvement is only in substitution of $\ln (1 / \varepsilon)$ with $\ln \ln (1 / \varepsilon)$ comparing to Rudolf's bound.

Our proof is also based on a random choice of points. A standard way to use randomness is to show that a certain "good" event $\mathcal{E}$ holds with a non-zero 
probability. Equivalently, one needs to show that the complement of $\mathcal{E}$, the event $\mathcal{E}^{c}$, holds with small probability. In order to do that, one tries to cover $\mathcal{E}^{c}$ by certain events, called individual events, to obtain good bounds on probabilities of individual events, and then to use the union bound. In this scheme one needs to have a good balance between (small) probabilities of individual events and the (large but not too large) size of the covering set. Since we need to prove that there exists a set $P$ of $n$ points such that there is no rectangle of volume $\varepsilon$ without a point from $P$, the natural idea would be to construct a finite set $\mathcal{N}$ of rectangles having reasonably large volume and such that property "each rectangle in $\mathcal{N}$ contains a point from $P$ " implies the property "each rectangle in $\mathcal{R}_{d}$ of volume at least $\varepsilon$ contains a point from $P$." In the case of uniform distribution on the cube $Q_{d}$, that is, in the case when the set $P$ consists of $N$ points independently drawn from the uniform distribution, an individual bound, that is, a bound on the event that a given box $B \in \mathcal{N}$ contains a point from $P$, is simply given by the volume of $B$, therefore the main difficulty is to construct the set $\mathcal{N}$ of not too large cardinality. Rudolf used the concept of $\delta$-cover [10, 4] to construct $\mathcal{N}$ and to estimate its size. We introduce the notion of $\delta$-net (see Definition 2.1), which fits better for random procedure described above and allows to obtain better bounds on its size, see Propositions 3.1 and 3.4 .

As usual in probabilistic proofs, we obtain the result with high probability. Very recently, Hinrichs, Krieg, Kunsch, and Rudolf [5] investigated the best bound that one can get using a random choice of points and showed that one cannot expect anything better than

$$
\max \left\{\frac{c}{\varepsilon} \ln \left(\frac{1}{\varepsilon}\right), \frac{d}{2 \varepsilon}\right\},
$$

where $c>0$ is an absolute constant. This in particular shows that our bounds are almost best possible for this method (up to $\ln d$ in the first estimate and up to $\ln \ln (1 / \varepsilon)$ in the second estimate).

In the case of large $\varepsilon$ we can improve the bound. The next theorem provides better bounds in the regime $\varepsilon \geq\left(\ln ^{2} d\right) /(d \ln \ln (2 d))$.

Theorem 1.2. There exists an absolute constant $C \geq 1$ such that the following holds. Let $d \geq 2$ and $\varepsilon \in(0,1 / 2]$ be such that $\varepsilon \geq \frac{\ln d}{d}$. Then

$$
N(\varepsilon, d) \leq \frac{C \ln d}{\varepsilon^{2}} \ln \left(\frac{1}{\varepsilon}\right) .
$$

This improves the Ullrich-Vybíral bound by removing one $\ln (1 / \varepsilon)$ factor. The proof of this theorem also uses random points uniformly distributed on the cube $Q_{d}$, however, as Hinrichs-Krieg-Kunsch-Rudolf's result shows, one cannot expect a bound better than $d / \varepsilon$, therefore one needs to adjust the distribution of the points. 
One way to adjust randomness was suggested by Sosnovec and then improved by Ullrich and Vybíral. They substituted the uniform distribution on the cube by a uniform distribution on a certain lattice inside the cube. This led to the logarithmic in $d$ upper bound (by the price of an additional factor $1 / \varepsilon$ ). Careful analysis of their proofs in comparison with Rudolf's proof shows that the main advantage of the use of a lattice is that the points on the lattice are $\varepsilon$-separated from the boundary of the cube. This leads to our adjustment of the uniform distribution on the cube - if a uniformly distributed over the cube random point falls too close to the boundary we slightly shift it to the interior, to ensure that it is $\varepsilon$-separated from the boundary. In the next section we introduce the function $\phi_{\varepsilon}$, which serves this purpose. Unfortunately, the size of $\delta$-nets is still too large, to deal with large $\varepsilon$, so we additionally introduce the notion of dinets - nets in the sense of dispersion (see Definition 2.2), which allows us to reduce the cardinality of a covering set (see Proposition 3.6) and hence to apply the union bound.

The upper bonds for $\varepsilon \leq 1 / 4$ from Theorems 1.1 and 1.2 are summarized in

$$
N(\varepsilon, d) \leq \begin{cases}\frac{C \ln d}{\varepsilon^{2}} \ln \left(\frac{1}{\varepsilon}\right), & \text { if } \varepsilon \geq \frac{\ln ^{2} d}{d \ln \ln (2 d)}, \\ \frac{C d}{\varepsilon} \ln \ln \left(\frac{1}{\varepsilon}\right), & \text { if } \frac{\ln ^{2} d}{d \ln \ln (2 d)} \geq \varepsilon \geq e^{-d}, \\ \frac{C \ln d}{\varepsilon} \ln \left(\frac{1}{\varepsilon}\right), & \text { if } e^{-d} \geq \varepsilon \geq \exp \left(-C^{d}\right), \\ \frac{C^{d}}{\varepsilon}, & \text { if } \varepsilon \leq \exp \left(-C^{d}\right)\end{cases}
$$

or in the following picture showing the corresponding regions.

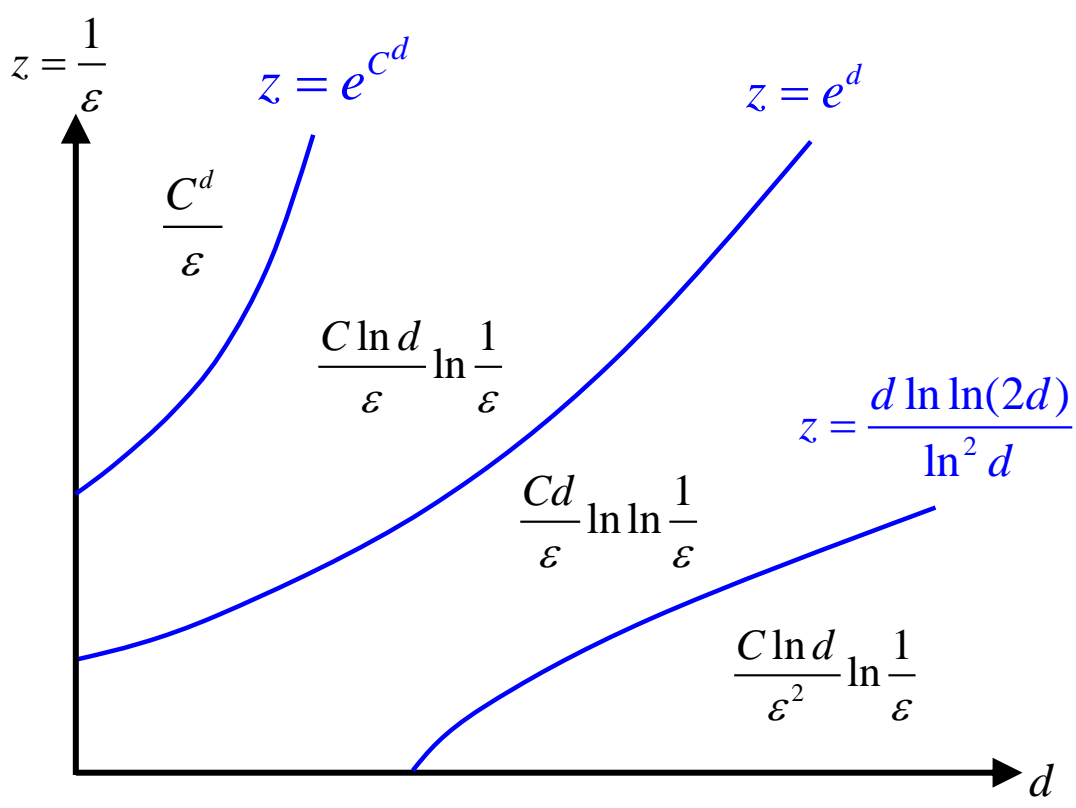


In terms of the minimal dispersion, Theorems 1.1 and 1.2 are equivalent to the following theorem.

Theorem 1.3. There exists an absolute constant $C \geq 1$ such that the following holds. Let $d \geq 2$ and $n \geq 2 \ln d$. Then

$$
\operatorname{disp}^{*}(n, d) \leq \frac{C \ln d}{n} \ln \left(\frac{n}{\ln d}\right), \quad \text { provided that } \quad n \geq e^{d} d \ln d,
$$

(ii) $\quad \operatorname{disp}^{*}(n, d) \leq \frac{C d}{n} \ln \ln \left(\frac{n}{d}\right), \quad$ provided that $\quad \frac{d^{2} \ln ^{2} \ln d}{\ln ^{2} d} \leq n \leq e^{d} d \ln d$,

(iii) $\quad \operatorname{disp}^{*}(n, d) \leq\left(\frac{C \ln d}{n} \ln \left(\frac{n}{\ln d}\right)\right)^{1 / 2}, \quad$ provided that $\quad n \leq \frac{d^{2} \ln ^{2} \ln d}{\ln ^{2} d}$.

Moreover, in the first two cases the random choice of points with respect to the uniform distribution on the cube $Q_{d}$ gives the result with high probability.

\subsection{Dispersion on the torus}

The corresponding dispersion on the torus can be described in terms of periodic axis parallel boxes. We denote such a set by $\widetilde{\mathcal{R}}_{d}$, that is

$$
\widetilde{\mathcal{R}}_{d}:=\left\{\prod_{i=1}^{d} I_{i}(a, b) \mid a, b \in Q_{d}\right\}
$$

where

$$
I_{i}(a, b):= \begin{cases}\left(a_{i}, b_{i}\right), & \text { whenever } 0 \leq a_{i}<b_{i} \leq 1, \\ {[0,1] \backslash\left[b_{i}, a_{i}\right],} & \text { whenever } 0 \leq b_{i}<a_{i} \leq 1 .\end{cases}
$$

The dispersion of a finite set $P \subset Q_{d}$ on the torus, the minimal dispersion on the torus, and its inverse are defined in the same way as above, but using sets from $\widetilde{\mathcal{R}}_{d}$, that is

$$
\widetilde{\operatorname{disp}}(P)=\sup \left\{|B| \mid B \in \widetilde{\mathcal{R}}_{d}, B \cap P=\emptyset\right\}, \quad \widetilde{\operatorname{disp}}^{*}(n, d)=\sup _{|P|=n} \widetilde{\operatorname{disp}}(P),
$$

and

$$
\widetilde{N}(\varepsilon, d)=\min \left\{n \in \mathbb{N} \mid \widetilde{\operatorname{disp}}^{*}(n, d) \leq \varepsilon\right\}
$$

It is known that

$$
\frac{d}{\varepsilon} \leq \tilde{N}(\varepsilon, d) \leq \frac{8 d}{\varepsilon}\left(\ln d+\ln \left(\frac{8}{\varepsilon}\right)\right)
$$

where the lower bound was proved by Ullrich [13] and the upper bound is due to Rudolf [10] (since there are no good bounds on the VC dimension of $\widetilde{\mathcal{R}}_{d}$, results 
of [2] are not directly applicable here). We would like to emphasize that contrary to the non-periodic case, even in the case of large $\varepsilon$, the lower bound is at least $d$. We improve the Rudolf upper bound in the case $\varepsilon \leq 1 / d$.

Theorem 1.4. There exists an absolute constant $C \geq 1$ such that the following holds. Let $d \geq 2$ and $\varepsilon \in(0,1 / 2]$. Then

$$
\begin{array}{ll}
\tilde{N}(\varepsilon, d) \leq \frac{C \ln d}{\varepsilon} \ln \left(\frac{1}{\varepsilon}\right), & \text { provided that } \varepsilon \leq \exp (-d), \\
\tilde{N}(\varepsilon, d) \leq \frac{C d \ln d}{\varepsilon}, & \text { provided that } \varepsilon \geq \exp (-d) .
\end{array}
$$

Moreover, the random choice of points with respect to the uniform distribution on the cube $Q_{d}$ gives the result with high probability. Equivalently, for $d \geq 2$ and $n \geq 2 d \ln d$ we have

$$
\widetilde{\operatorname{disp}}^{*}(n, d) \leq \frac{C \ln d}{n} \ln \left(\frac{n}{\ln d}\right), \quad \text { provided that } \quad n \geq e^{d} d \ln d,
$$

$$
\widetilde{\operatorname{disp}}^{*}(n, d) \leq \frac{C d \ln d}{n}, \quad \quad \text { provided that } \quad 2 d \ln d \leq n \leq e^{d} d \ln d .
$$

Our bound on $\widetilde{N}(\varepsilon, d)$ reduces the factor $d$ in Rudolf's estimate to ln $d$ in the case when $\varepsilon \leq \exp (-d)$ and removes the summand $\ln (1 / \varepsilon)$ if $\exp (-d)<\varepsilon \leq 1 / d$. However, if $\varepsilon \geq 1 / d$, it gives the same order $(d \ln d) / \varepsilon$.

The proof is the same as for Theorem 1.1, using random points and a $\delta$-net constructed for periodic boxes. Unfortunately, in the construction of nets for the second bound in Theorem 1.1 and for the bound in Theorem 1.2, we essentially use that boxes are not periodic and therefore the construction cannot be extended to the periodic case (for Theorem 1.2 it is also clear in view of the Ullrich lower bound on $\widetilde{N}(\varepsilon, d)$ ). We would also like to note that the Hinrichs-Krieg-Kunsch-Rudolf's result on best possible lower bound (3) which may be obtained by using random points uniformly distributed on the cube holds for the periodic setting as well, therefore the factor $\ln (1 / \varepsilon)$ in our first estimate is unavoidable by this method. In the second case, $\varepsilon \geq \exp (-d)$, we have $\ln (1 / \varepsilon) \leq d$, so there is a hope to remove $\ln d$ factor and to obtain the best possible estimate, on the other hand it is possible that the bound is the best possible for this method.

\section{Nets, dinets, and a probability lemma}

We need more notations. Given a positive integer $m$ we denote $[m]=\{1,2, \ldots, m\}$. Given $\varepsilon>0$, we consider sets of (periodic) axis parallel of volume at least $\varepsilon$,

$$
\mathcal{B}_{\varepsilon, d}:=\left\{B \in \mathcal{R}_{d}|| B \mid \geq \varepsilon\right\} \quad \text { and } \quad \widetilde{\mathcal{B}}_{\varepsilon, d}:=\left\{B \in \widetilde{\mathcal{R}}_{d}|| B \mid \geq \varepsilon\right\} .
$$


We introduce the following definition.

Definition 2.1 ( $\delta$-net for $\left.\mathcal{B}_{\varepsilon, d}\right)$. Given $\varepsilon, \delta>0$ we say that $\mathcal{N} \subset \mathcal{R}_{d}$ is a $\delta$-net for $\mathcal{B}_{\varepsilon, d}$ if for every $B \in \mathcal{B}_{\varepsilon, d}$ there exists $B_{0} \in \mathcal{N}$ such that $B_{0} \subset B$ and

$$
\left|B_{0}\right| \geq(1-\delta)|B| \text {. }
$$

We define a $\delta$-net for $\widetilde{\mathcal{B}}_{\varepsilon, d}$ in a similar way.

To deal with large $\varepsilon$ with respect to the dimension, say when $\varepsilon \geq 1 / d$, we adjust the definition of a $\delta$-net by introducing the notion of $\delta$-dinet-a $\delta$-net in $a$ sense of dispersion. The key idea leading to this approach is an observation that we do not need to consider points which are too close to the boundary of the cube $Q_{d}$. As we mentioned in the introduction, this idea was already implicitly used in [11, 15]. First given $\varepsilon \in(0,1 / 2)$ define an auxiliary function $\phi_{\varepsilon}:[0,1] \rightarrow[\varepsilon, 1-\varepsilon]$ by

$$
\phi_{\varepsilon}(t)= \begin{cases}\varepsilon & \text { if } 0 \leq t<\varepsilon \\ t & \text { if } \varepsilon \leq t \leq 1-\varepsilon \\ 1-\varepsilon & \text { if } 1-\varepsilon<t \leq 1\end{cases}
$$

Given $x \in Q_{d}$ we also write $\phi_{\varepsilon}(x)$ for $\left\{\phi_{\varepsilon}\left(x_{i}\right)\right\}_{i=1}^{d}$.

Definition $2.2\left(\delta\right.$-dinet for $\left.\mathcal{B}_{\varepsilon, d}\right)$. Given $\varepsilon, \delta>0$ we say that $\mathcal{N} \subset \mathcal{R}_{d}$ is a $\delta$-dinet for $\mathcal{B}_{\varepsilon, d}$ if for every $B \in \mathcal{B}_{\varepsilon, d}$ there exists $B_{0} \in \mathcal{N}$ such that

$$
\left|B_{0}\right| \geq(1-\delta)|B|
$$

and such that for every $x \in Q_{d}$ the following implication holds

$$
x \in B_{0} \quad \Longrightarrow \quad \phi_{\varepsilon}(x) \in B
$$

Note that every $\delta$-dinet $\mathcal{N}$ for $\mathcal{B}_{\varepsilon, d}$ has the following property allowing to bound from above the number of points needed to have a given dispersion, namely, for every $n \geq 1$, every set of points $P=\left\{x_{1}, \ldots, x_{n}\right\} \subset Q_{d}$ the statement "each box from $\mathcal{N}$ contains at least one point from $P$ " implies the statement "each box from $\mathcal{B}_{\varepsilon, d}$ contains at least one point from $\phi_{\varepsilon}(P)$ ".

A variant of the following lemma using random points and the union bound was proved in [10] (see Theorem 1 there). We provide a proof for completeness.

Lemma 2.3. Let $d \geq 1$ and $\varepsilon, \delta \in(0,1)$. Let $\mathcal{N}$ be either a $\delta$-net for $\mathcal{B}_{\varepsilon, d}$ or a $\delta$-dinet for $\mathcal{B}_{\varepsilon, d}$ and let $\widetilde{\mathcal{N}}$ be a $\delta$-net for $\widetilde{\mathcal{B}}_{\varepsilon, d}$. Assume both $|\mathcal{N}| \geq 3$ and $|\tilde{\mathcal{N}}| \geq 3$. Then

$$
N(\varepsilon, d) \leq \frac{3 \ln |\mathcal{N}|}{(1-\delta) \varepsilon} \quad \text { and } \quad \tilde{N}(\varepsilon, d) \leq \frac{3 \ln |\tilde{\mathcal{N}}|}{(1-\delta) \varepsilon}
$$


Remark 2.4. As usual for proofs involving the union bound, our proof shows that the random choice of $N=\left\lfloor\frac{3 \ln |\mathcal{N}|}{(1-\delta) \varepsilon}\right\rfloor$ (resp. $N=\left\lfloor\frac{3 \ln |\widetilde{\mathcal{N}}|}{(1-\delta) \varepsilon}\right\rfloor$ ) points gives the result with high probability, more precisely with probability at least 1 $1 /|\mathcal{N}|$. In the case of $\delta$-nets the randomness is with respect to the independent uniform choice of points on $Q_{d}$, while in the case of $\delta$-dinets one needs to adjust the choice of independent uniformly distributed points by the function $\phi_{\varepsilon}$.

Proof. We show a proof for a $\delta$-net $\mathcal{N}$ for $\mathcal{B}_{\varepsilon, d}$, the other two cases are the same. Let $\mathcal{N}$ be a $\delta$-net for $\mathcal{B}_{\varepsilon, d}$. Consider $N$ independent random points $X_{1}$, ..., $X_{N}$ uniformly chosen from $Q_{d}$. By the definition of a $\delta$-net, it is enough to show that for every $B \in \mathcal{N}$ with $|B| \geq v:=(1-\delta) \varepsilon$ there exists $j \leq N$ such that $X_{j} \in B$. Fix such a box $B$. Using that the volume of $B$ is at least $v$ and the independence of $X_{j}$ 's, we obtain

$$
\mathbb{P}\left(\left\{\forall j \leq N: X_{j} \notin B\right\}\right) \leq(1-v)^{N}<\exp (-v N) .
$$

Therefore, by the union bound,

$$
\mathbb{P}\left(\left\{\exists B \in \mathcal{N}:|B| \geq v \quad \text { and } \quad \forall j \leq N: \quad X_{j} \notin B\right\}\right)<|\mathcal{N}| \exp (-v N) .
$$

Thus, as far as $|\mathcal{N}| \exp (-v N) \leq 1$, there exists a realization of $X_{j}$ 's with the desired property. Moreover, if $N=\left\lfloor\frac{3 \ln |\mathcal{N}|}{(1-\delta) \varepsilon}\right\rfloor$ then the "good" probability is $1-|\mathcal{N}| \exp (-v N) \geq 1-1 /|\mathcal{N}|$. This implies both Lemma 2.3 and Remark 2.4.

\section{Cardinality of nets}

As is seen from Lemma 2.3 and Remark 2.4, to prove our theorems, it is enough to construct nets of not so large cardinality. The next simple observation is one of key ideas in our estimates. Let $\varepsilon>0$ and let $\ell_{1}, \ldots, \ell_{d}>0$ be such that

$$
\prod_{i=1}^{d} \ell_{i} \geq \varepsilon .
$$

Denote by $\sigma=\sigma\left(\ell_{1}, \ldots, \ell_{d}\right)$ a permutation such that

$$
\ell_{\sigma(1)} \leq \ell_{\sigma(2)} \leq \ldots \leq \ell_{\sigma(d)}
$$


(for each sequence we fix one such permutation). Then for every $j \geq 1$ we clearly have

$$
\ell_{\sigma(j)} \geq\left(\prod_{i=1}^{j} \ell_{\sigma(i)}\right)^{1 / j} \geq \varepsilon^{1 / j}>1-\frac{\ln (1 / \varepsilon)}{j} .
$$

A naive approach to approximate rectangles from $\mathcal{B}_{\varepsilon, d}$ is to say that given a rectangle $B=\prod_{i=1}^{d} I_{i} \in \mathcal{B}_{\varepsilon, d}$ the smallest length $\ell_{i}=\left|I_{i}\right|$ is at least $\varepsilon$. Therefore, we can take $(1 /(4 \varepsilon))$-net $\mathcal{M}$ in $[0,1]$ and approximate each $I_{i}$ with segments having endpoints in $\mathcal{M}$. This approach would lead to a net of the order $(1 /(4 \varepsilon))^{2 d}$, which is not acceptable for our purpose (this would also lead to a huge loss in volume, but already the size of a net is too large). Instead, we use formula (5), to say that the larger $i$ the coarser net in $[0,1]$ is needed in order to approximate the corresponding interval $I_{\sigma(i)}$. Of course, simultaneously, we need to control the loss in volume in our approximation. The next proposition utilizes this idea. It works for both the periodic and non-periodic settings. Since we will be using this result in several dimensions, it would be convenient to formulate it for boxes in $\mathbb{R}^{m}$.

Proposition 3.1. Let $m \geq 2$ be an integer and $\varepsilon \in(0,1)$. There are $(1 / 2)$ nets $\mathcal{N}$ and $\widetilde{\mathcal{N}}$ for $\mathcal{B}_{\varepsilon, m}$ and $\widetilde{\mathcal{B}}_{\varepsilon, m}$ respectively, each of them of cardinality at most

$$
\frac{(14 m)^{4 m}}{\varepsilon^{2 \log _{2}(2 m)}}
$$

Remark 3.2. If $m=2^{k}$ for some integer $k$ then our proof gives slightly better estimate, namely

$$
\frac{(24 m)^{2 m}}{\varepsilon^{2 \log _{2} m}} .
$$

Remark 3.3. Clearly, Lemma 2.3 and Remark 2.4 combined with this proposition (applied with $m=d$ ) yield Theorem 1.4 as well as the first bound in Theorem 1.1.

Proof. The construction of nets in $\mathcal{B}_{\varepsilon, m}$ and $\widetilde{\mathcal{B}}_{\varepsilon, m}$ are essentially the same. We provide a proof for a net in $\widetilde{\mathcal{B}}_{\varepsilon, m}$, since the proof for a net in $\mathcal{B}_{\varepsilon, m}$ is somewhat easier - we do not need to consider intervals $I_{i}(a, b)$ with $a_{i}>b_{i}$.

Fix $k \geq 1$ such that $2^{k} \leq m<2^{k+1}$. Fix a partition of $[m]$ into $k+1$ disjoint sets $A_{1}, \ldots, A_{k+1}$ with $\left|A_{1}\right|=2,\left|A_{k+1}\right|=m-2^{k}$ (this set is empty if $m=2^{k}$ ), and $\left|A_{j}\right|=2^{j-1}$ for $2 \leq j \leq k$. For $j \leq k+1$ denote

$$
\delta^{(j)}=2^{-k-3} \varepsilon^{2^{1-j}} \quad \text { and } \quad D_{j}=\left\{0, \delta^{(j)}, 2 \delta^{(j)}, \ldots, s_{j} \delta^{(j)}\right\},
$$


where $s_{j}=\left\lfloor 1 / \delta^{(j)}\right\rfloor$ (note that dealing with $\mathcal{B}_{\varepsilon, m}$ we do not need to have 0 in $\left.D_{j}\right)$.

We are now ready to define a part of our net corresponding to this partition of $[m]$ as the set

$\mathcal{N}_{*}\left(A_{1}, \ldots, A_{k+1}\right):=\left\{\prod_{i=1}^{m} I_{i}(x, y) \mid x, y \in Q_{m}, \forall j \leq k+1 \forall i \in A_{j}: x_{i} \neq y_{i} \in D_{j}\right\}$.

Then the cardinality of this set can be estimated as

$$
\begin{aligned}
\left|\mathcal{N}_{*}\left(A_{1}, \ldots, A_{k+1}\right)\right| & \leq \prod_{j=1}^{k+1} \prod_{i \in A_{j}}\left|D_{j}\right|\left(\left|D_{j}\right|-1\right) \leq \prod_{j=1}^{k+1} \prod_{i \in A_{j}} \frac{2}{\left(\delta^{(j)}\right)^{2}} \leq 2^{m} \prod_{j=1}^{k+1} \prod_{i \in A_{j}} \frac{4^{k+3}}{\varepsilon^{2^{2-j}}} \\
& \leq 2^{m}\left(\frac{4^{k+3}}{\varepsilon^{2}}\right)^{2} \prod_{j=2}^{k+1}\left(\frac{4^{k+3}}{\varepsilon^{2^{2-j}}}\right)^{2^{j-1}}=2^{m} \frac{4^{2^{k+1}(k+3)}}{\varepsilon^{2(k+1)}} \leq \frac{2^{m}\left(64 m^{2}\right)^{2 m}}{\varepsilon^{2 \log _{2}(2 m)}}
\end{aligned}
$$

(note that if $m=2^{k}$, then the set $A_{k+1}$ is empty and $j$ runs between 1 and $k$, which leads to the bound from Remark 3.2 .

To complete the construction, we take the union over all partitions of $[\mathrm{m}]$ into such sets $A_{1}, \ldots, A_{k+1}$,

$$
\mathcal{N}:=\bigcup \mathcal{N}_{*}\left(A_{1}, \ldots, A_{k+1}\right) .
$$

The number of partitions can be estimated as

$$
\left(\begin{array}{c}
m \\
2^{k}
\end{array}\right)\left(\begin{array}{c}
2^{k} \\
2^{k-1}
\end{array}\right)\left(\begin{array}{c}
2^{k-1} \\
2^{k-2}
\end{array}\right) \cdots\left(\begin{array}{l}
4 \\
2
\end{array}\right) \leq 2^{2 m}
$$

hence

$$
|\mathcal{N}| \leq \frac{2^{3 m}(8 m)^{4 m}}{\varepsilon^{2 \log _{2}(2 m)}} \leq \frac{(14 m)^{4 m}}{\varepsilon^{2 \log _{2}(2 m)}}
$$

It remains to show that $\mathcal{N}$ is indeed a $(1 / 2)$-net for $\widetilde{\mathcal{B}}_{\varepsilon, m}$. Let $a, b \in Q_{m}$ and $B=\prod_{i=1}^{m} I_{i}(a, b)$ be of volume at least $\varepsilon$. For $i \leq m$ let $\ell_{i}$ be the length of $I_{i}(a, b)$. Let $\sigma=\sigma\left(\ell_{1}, \ldots, \ell_{m}\right)$ be the permutation defined by (4). Consider the following partitions of $[m]$,

$A_{1}^{\sigma}=\sigma(\{1,2\}), \quad A_{k+1}^{\sigma}=\sigma\left(\left\{2^{k}+1, \ldots, m\right\}\right), \quad$ and $\quad A_{j}^{\sigma}=\sigma\left(\left\{2^{j-1}+1, \ldots, 2^{j}\right\}\right)$,

$2 \leq j \leq k$, and note that by (5) for every $j \leq k+1$ and every $i \in A_{j}^{\sigma}$ one has

$$
\left|I_{i}(a, b)\right|=\ell_{i} \geq \ell_{\sigma\left(2^{j-1}\right)} \geq \varepsilon^{1 / 2^{j-1}}=2^{k+3} \delta^{(j)} .
$$


Take a box $B_{0}=\prod_{i=1}^{m} I_{i}(x, y)$ from $\mathcal{N}_{*}\left(A_{1}^{\sigma}, \ldots, A_{k+1}^{\sigma}\right)$ such that for every $j \leq k+1$ and every $i \in A_{j}^{\sigma}$ one has

$$
a_{i} \leq x_{i}, \quad b_{i} \geq y_{i}, \quad x_{i}-a_{i} \leq \delta^{(j)}, \quad \text { and } \quad b_{i}-y_{i} \leq \delta^{(j)}
$$

(if $a_{i}>s_{j} \delta^{(j)}$ we take $x_{i}=0$ ). The lower bound $(6)$ on the length of $I_{i}(a, b)$ implies that $I_{i}(x, y) \subset I_{i}(a, b)$. Thus, $B_{0} \subset B$ and, using (6) again,

$$
\begin{aligned}
\left|B_{0}\right| & =\prod_{j=1}^{k+1} \prod_{i \in A_{j}^{\sigma}}\left|I_{i}(x, y)\right| \geq \prod_{j=1}^{k+1} \prod_{i \in A_{j}^{\sigma}}\left(\ell_{i}-2 \delta^{(j)}\right)=\prod_{i=1}^{m} \ell_{i} \prod_{j=1}^{k+1} \prod_{i \in A_{j}^{\sigma}}\left(1-\frac{2 \delta^{(j)}}{\ell_{i}}\right) \\
& \geq|B| \prod_{j=1}^{k+1}\left(1-\frac{2 \delta^{(j)}}{\ell_{\sigma\left(2^{j-1}\right)}}\right)^{\left|A_{j}^{\sigma}\right|} \geq|B| \prod_{j=1}^{k+1}\left(1-\frac{1}{2^{k+2}}\right)^{\left|A_{j}^{\sigma}\right|} \geq|B|\left(1-\frac{1}{2^{k+2}}\right)^{m} \\
& \geq|B|\left(1-\frac{1}{2 m}\right)^{m} \geq \frac{1}{2}|B| .
\end{aligned}
$$

This completes the proof.

Next we show how to improve the bound of Proposition 3.1 for nonperiodic boxes in the case when $\varepsilon$ is not very small with respect to dimension, say, when $4 \ln (1 / \varepsilon) \leq d$. The key observation here is that in the case $4 \ln (1 / \varepsilon) \leq d$ a rectangle $B=\prod_{i=1}^{d} I_{i} \in \mathcal{B}_{\varepsilon, d}$ has many intervals $I_{i}$ of length close to one, namely, by (5), $\left|I_{\sigma(i)}\right| \geq 1-1 / L$ whenever $i \geq L \ln (1 / \varepsilon)$. For such an interval we do not need to take a net in $[0,1]$ in order to approximate the end points - it is enough to approximate the left end point by a net in $[0,1 / L]$ and the right end point by a net in $[1-1 / L, 1]$. This leads to a significant improvement in the size of the net. Of course, this approach cannot work for periodic boxes.

Proposition 3.4. Let $d \geq 4$ be an integer, $\varepsilon \in(0,1 / 4]$ and assume that $d \geq 4 \ln (1 / \varepsilon)$. Then $\mathcal{B}_{\varepsilon, d}$ admits a $(3 / 4)$-net of cardinality at most

$$
\exp (C d \ln \ln (1 / \varepsilon))
$$

where $C \geq 1$ is an absolute constant.

Remark 3.5. Clearly, Lemma 2.3 and Remark 2.4 combined with this proposition yield the second bound in Theorem 1.1 . 
Proof. The proof is similar to the proof of Proposition 3.1, but we deal more carefully with the approximation of long segments.

Set $k$ to be the smallest integer such that $2^{k} \geq 2 \ln (1 / \varepsilon)$ and let $m=2^{k}$. Clearly, $k \geq 1, m \geq 2$. Then $d \geq 4 \ln (1 / \varepsilon)>m$. Fix an integer $n \geq k$ such that $2^{n} \leq d<2^{n+1}$. Fix a partition of $[d]$ into $n-k+2$ disjoint sets $A_{0}, \ldots$, $A_{n-k+1}$ with $\left|A_{0}\right|=m,\left|A_{n-k+1}\right|=d-2^{n}$ (this set is empty if $d=2^{n}$ ), and $\left|A_{j}\right|=2^{k+j-1}$ for $1 \leq j \leq n-k$. Denote

$$
\delta=\frac{1}{8 d}, \quad D_{1}=\{\delta, 2 \delta, \ldots, s \delta\}, \quad \text { and } \quad D_{2}=\{1-\delta, 1-2 \delta, \ldots, 1-s \delta\},
$$

where $s_{j}=\lfloor 1 / \delta\rfloor$.

Next, for every $1 \leq j \leq n-k+1$ we consider the set $P_{j} \subset D_{1} \times D_{2}$ of all pairs $(p, q)$ satisfying $p \in D_{1}, q \in D_{2}, p<q$, and

$$
p \leq 2^{1-k-j} \ln (1 / \varepsilon)+\delta \quad \text { and } \quad q \geq 1-2^{1-k-j} \ln (1 / \varepsilon)-\delta .
$$

Using $2^{k} \geq 2 \ln (1 / \varepsilon)$ and $\delta=1 /(8 d)$, we observe that the cardinality of $P_{j}$ is

$$
\left|P_{j}\right| \leq\left(\frac{2^{1-k-j} \ln (1 / \varepsilon)}{\delta}+1\right)^{2} \leq\left(\frac{8 d}{2^{j}}+1\right)^{2} \leq \frac{d^{2}}{4^{j-2}}
$$

Let $\mathcal{N}_{0}\left(A_{0}\right)$ be the $(1 / 2)$-net of cardinality at most

$$
n_{0}:=\frac{(24 m)^{2 m}}{\varepsilon^{2 \log _{2} m}}
$$

for $\mathcal{B}_{\varepsilon, m}$ from Proposition 3.1 constructed in $\mathbb{R}^{A_{0}}$ (see also Remark 3.2). Let $\mathcal{N}_{*}=\mathcal{N}_{*}\left(A_{0}, \ldots, A_{n-k+1}\right)$ be the set of all boxes $\prod_{i=1}^{d}\left[x_{i}, y_{i}\right)$ such that

$$
\prod_{i \in A_{0}}\left[x_{i}, y_{i}\right) \in \mathcal{N}_{0}\left(A_{0}\right)
$$

and for every $1 \leq j \leq n-k+1$ and for every $i \in A_{j}$ the pair $\left(x_{i}, y_{i}\right) \in P_{j}$. Then, using $2^{n} \leq d<2^{n+1}$ and $m=2^{k}$, the cardinality of $\mathcal{N}_{*}$ can be estimated as

$$
\begin{aligned}
\left|\mathcal{N}_{*}\right| & \leq\left|\mathcal{N}_{0}\left(A_{0}\right)\right| \prod_{j=1}^{n-k+1} \prod_{i \in A_{j}}\left|P_{j}\right| \leq n_{0} \prod_{j=1}^{n-k+1}\left(\frac{d^{2}}{4^{j-2}}\right)^{\left|A_{j}\right|} \leq n_{0} \prod_{j=1}^{n-k+1} \frac{d^{2^{k+j}}}{4^{(j-2) 2^{k+j-1}}} \\
& \leq n_{0} \frac{d^{2^{n+2}}}{4^{(n-k-3) 2^{n+1}}}=n_{0}\left(\frac{4^{k+4} d^{2}}{4^{n+1}}\right)^{2^{n+1}} \leq n_{0}\left(4^{4} m^{2}\right)^{2 d}=\frac{(24 m)^{2 m}}{\varepsilon^{2 \log _{2} m}}(16 m)^{4 d} .
\end{aligned}
$$


Finally we define our net as the union over all partitions of $[d]$ into such sets $A_{0}, \ldots, A_{n-k+1}$,

$$
\mathcal{N}:=\bigcup \mathcal{N}_{*}\left(A_{0}, \ldots, A_{n-k+1}\right) .
$$

The number of partitions can be estimated as

$$
\left(\begin{array}{c}
d \\
2^{n}
\end{array}\right)\left(\begin{array}{c}
2^{n} \\
2^{n-1}
\end{array}\right)\left(\begin{array}{c}
2^{n-1} \\
2^{n-2}
\end{array}\right) \cdots\left(\begin{array}{c}
2^{k+1} \\
2^{k}
\end{array}\right) \leq 2^{2 d-2^{k+1}} \leq 2^{2 d-2 m}
$$

hence

$$
|\mathcal{N}| \leq 2^{2 d-2 m} \frac{(24 m)^{2 m}}{\varepsilon^{2 \log _{2} m}}(16 m)^{4 d} \leq \frac{(12 m)^{2 m}}{\varepsilon^{2 \log _{2} m}}(24 m)^{4 d} \leq \frac{(24 m)^{6 d}}{\varepsilon^{2 \log _{2} m}} .
$$

Using that $m \leq 4 \ln (1 / \varepsilon) \leq d$, we obtain

$$
|\mathcal{N}| \leq \exp \left(6 d \ln (24 m)+2\left(\log _{2} m\right)(\ln (1 / \varepsilon)) \leq \exp (C d \ln \ln (1 / \varepsilon)),\right.
$$

where $C \geq 1$ is an absolute constant.

It remains to show that $\mathcal{N}$ is indeed a $(3 / 4)$-net for $\mathcal{B}_{\varepsilon, d}$. Let $a, b \in Q_{d}$ with $a_{i}<b_{i}$ for all $i \leq d$, and $B=\prod_{i=1}^{d}\left[a_{i}, b_{i}\right)$ be of volume at least $\varepsilon$. For $i \leq d$ let $\ell_{i}=b_{i}-a_{i}$. Let $\sigma=\sigma\left(\ell_{1}, \ldots, \ell_{d}\right)$ be the permutation defined by (4). Consider the following partitions of $[d]$,

$A_{0}^{\sigma}=\sigma([m]), \quad A_{n-k+1}^{\sigma}=\sigma\left(\left\{2^{n}+1, \ldots, d\right\}\right), \quad$ and $\quad A_{j}^{\sigma}=\sigma\left(\left\{2^{k+j-1}+1, \ldots, 2^{k+j}\right\}\right)$,

$1 \leq j \leq n-k$.

Fix for a moment $1 \leq j \leq n-k+1$ and $i \in A_{j}^{\sigma}$. Using $2^{k} \geq 2 \ln (1 / \varepsilon)$ and (5), we observe that

$$
b_{i}-a_{i}=\ell_{i} \geq \ell_{\sigma\left(2^{k+j-1}\right)}>1-\frac{\ln (1 / \varepsilon)}{2^{k+j-1}} \geq 1-2^{-j} .
$$

Take a pair $\left(x_{i}, y_{i}\right) \in D_{1} \times D_{2}$ satisfying

$$
a_{i} \leq x_{i}, \quad b_{i} \geq y_{i}, \quad x_{i}-a_{i} \leq \delta, \quad \text { and } \quad b_{i}-y_{i} \leq \delta .
$$

Then $y_{i}-x_{i} \geq b_{i}-a_{i}-2 \delta>1-2^{-j}-2 \delta>0$ and

$$
y_{i} \geq b_{i}-\delta>1-\frac{\ln (1 / \varepsilon)}{2^{k+j-1}}-\delta \quad \text { and } \quad x_{i} \leq a_{i}+\delta<\frac{\ln (1 / \varepsilon)}{2^{k+j-1}}+\delta,
$$

in other words the pair $\left(x_{i}, y_{i}\right) \in P_{j}$. 
Consider the box $B_{0}=\prod_{i=1}^{d}\left[x_{i}, y_{i}\right)$ such that for every $1 \leq j \leq n-k+1$ and every $i \in A_{j}^{\sigma}$ the pair $\left(x_{i}, y_{i}\right)$ is constructed as above and where

$$
B_{0}^{\prime}=\prod_{i \in A_{0}^{\sigma}}\left[x_{i}, y_{i}\right) \in \mathcal{N}_{0}\left(A_{0}^{\sigma}\right) \quad \text { approximates } \quad B^{\prime}=\prod_{i \in A_{0}^{\sigma}}\left[a_{i}, b_{i}\right)
$$

as in Proposition 3.1 (note that $m$-dimensional volume of $B^{\prime}$ is at least $\varepsilon$, so $\left.B^{\prime} \in \mathcal{B}_{\varepsilon, m}\right)$. Then by construction $B_{0} \in \mathcal{N}_{*}\left(A_{1}^{\sigma}, \ldots, A_{k+1}^{\sigma}\right), B_{0} \subset B$, and

$$
\left|B_{0}^{\prime}\right| \geq \frac{1}{2}\left|B^{\prime}\right|=\frac{1}{2} \prod_{i \in A_{0}^{\sigma}} \ell_{i} .
$$

Furthermore, using $\delta=1 /(8 d)$ and the bound (7) again,

$$
\begin{aligned}
\left|B_{0}\right| & =\left|B_{0}^{\prime}\right| \prod_{j=1}^{n-k+1} \prod_{i \in A_{j}^{\sigma}}\left|y_{i}-x_{i}\right| \geq \frac{1}{2} \prod_{i \in A_{0}^{\sigma}} \ell_{i} \prod_{j=1}^{n-k+1} \prod_{i \in A_{j}^{\sigma}}\left(\ell_{i}-2 \delta\right) \\
& =\frac{1}{2} \prod_{i=1}^{d} \ell_{i} \prod_{j=1}^{n-k+1} \prod_{i \in A_{j}^{\sigma}}\left(1-\frac{2 \delta}{\ell_{i}}\right) \geq \frac{1}{2}|B| \prod_{j=1}^{n-k+1}\left(1-\frac{2 \delta}{1-2^{-j}}\right)^{\left|A_{j}^{\sigma}\right|} \\
& \geq \frac{1}{2}|B|\left(1-\frac{1}{2 d}\right)^{d-2^{n}} \prod_{j=1}^{n-k}\left(1-\frac{1}{2 d}\right)^{2^{k+j-1}} \geq \frac{1}{2}|B|\left(1-\frac{1}{2 d}\right)^{d} \geq \frac{1}{4}|B| .
\end{aligned}
$$

This completes the proof.

Finally, we want to improve bounds in the case of large $\varepsilon$. The following proposition is an almost immediate consequence of Proposition 3.1 and definitions. The key observation here is also the fact that a rectangle $B=\prod_{i=1}^{d} I_{i} \in \mathcal{B}_{\varepsilon, d}$ has many intervals $I_{i}$ of the length close to one, but now they will be so close to one, that we can substitute them just by $[0,1]$. More precisely, using our function $\phi_{\varepsilon}$, if the length of $I_{i}$ is at least $1-\varepsilon$ then for every $z \in[0,1]$ one has $\phi_{\varepsilon}(z) \in I_{i}$, hence we do not need to approximate such intervals. This leads to our definition of a dinet and to better bounds of cardinality of dinets versus regular nets. Unfortunately, this also leads to an additional factor $1 / \varepsilon$ in the final bound. As in the previous proposition, such an approach essentially uses that we are in the non-periodic setting.

Proposition 3.6. Let $d \geq 4$ be an integer, $\varepsilon \in(0,1 / 2]$ and assume that $d \geq(\ln (1 / \varepsilon)) / \varepsilon$. There is a $(1 / 2)$-dinet $\mathcal{N}$ for $\mathcal{B}_{\varepsilon, d}$ of cardinality at most

$$
\exp \left(\frac{9 \ln (1 / \varepsilon) \ln (18 d)}{\varepsilon}\right) \text {. }
$$


Remark 3.7. Clearly, Lemma 2.3 combined with this proposition yields Theorem 1.2. We can also use Remark 2.4 to claim that a random choice of points works with high probability, but here the randomness will be with respect to the uniform distribution on the cube adjusted by the function $\phi_{\varepsilon}$.

Proof. Fix the smallest integer $m \geq(\ln (1 / \varepsilon)) / \varepsilon$. Given subset $A \subset[d]$ of cardinality $m$, let $\mathcal{N}_{0}(A)$ be the $(1 / 2)$-net of cardinality at most

$$
n_{0}:=\frac{(14 m)^{4 m}}{\varepsilon^{2 \log _{2}(2 m)}}
$$

for $\mathcal{B}_{\varepsilon, m}$ from Proposition 3.1 constructed in $\mathbb{R}^{A}$. Let $\mathcal{N}_{*}(A)$ be the set of all boxes $\prod_{i=1}^{d}\left[x_{i}, y_{i}\right)$ such that

$$
\prod_{i \in A}\left[x_{i}, y_{i}\right) \in \mathcal{N}_{0}(A)
$$

and for every $i \notin A,\left[x_{i}, y_{i}\right)=[0,1)$. Let

$$
\mathcal{N}=\bigcup_{\substack{A \subset[d] \\|A|=m}} \mathcal{N}_{*}(A)
$$

Then the cardinality of $\mathcal{N}$ is at most

$$
\left(\begin{array}{c}
d \\
m
\end{array}\right) n_{0} \leq\left(\frac{e d}{m}\right)^{m} n_{0} \leq \frac{\left(14^{4} e m^{3} d\right)^{m}}{\varepsilon^{2 \log _{2}(2 m)}} \leq \frac{(18 d)^{4 m}}{\varepsilon^{2 \log _{2}(2 m)}} .
$$

Since $(\ln (1 / \varepsilon)) / \varepsilon \leq m \leq d$ and $m \leq 2(\ln (1 / \varepsilon)) / \varepsilon$, this implies

$$
|\mathcal{N}| \leq \exp \left(4 m \ln (18 d)+2 \log _{2}(2 m) \ln (1 / \varepsilon)\right) \leq \exp \left(\frac{9 \ln (1 / \varepsilon) \ln (18 d)}{\varepsilon}\right) .
$$

Now we show that $\mathcal{N}$ is a $(1 / 2)$-dinet for $\mathcal{B}_{\varepsilon, d}$. Let $a, b \in Q_{d}$ with $a_{i}<b_{i}$ for all $i \leq d$, and $B=\prod_{i=1}^{d}\left[a_{i}, b_{i}\right)$ be of volume at least $\varepsilon$. For $i \leq d$ let $\ell_{i}=b_{i}-a_{i}$. Let $\sigma=\sigma\left(\ell_{1}, \ldots, \ell_{d}\right)$ be the permutation defined by (4) and denote $A^{\sigma}=\sigma([m])$. Consider the box $B_{0}=\prod_{i=1}^{d}\left[x_{i}, y_{i}\right)$ such that $\left[x_{i}, y_{i}\right)=[0,1)$ for every $i \notin A^{\sigma}$ and

$$
B_{0}^{\prime}=\prod_{i \in A^{\sigma}}\left[x_{i}, y_{i}\right) \in \mathcal{N}_{0}\left(A^{\sigma}\right) \quad \text { approximates } \quad B^{\prime}=\prod_{i \in A^{\sigma}}\left[a_{i}, b_{i}\right)
$$


as in Proposition 3.1 (note that $m$-dimensional volume of $B^{\prime}$ is at least $\varepsilon$, so $\left.B^{\prime} \in \mathcal{B}_{\varepsilon, m}\right)$. Then by construction $B_{0} \in \mathcal{N}_{*}\left(A^{\sigma}\right)$, and

$$
\left|B_{0}\right|=\left|B_{0}^{\prime}\right| \geq \frac{1}{2}\left|B^{\prime}\right|=\frac{1}{2} \prod_{i \in A^{\sigma}} \ell_{i} \geq \frac{1}{2}|B| .
$$

Finally assume that $z \in B_{0}$. If $i \notin A^{\sigma}$ then using (5) and $m \geq(\ln (1 / \varepsilon)) / \varepsilon$ we have

$$
b_{i}-a_{i}=\ell_{i} \geq \ell_{\sigma(m)}>1-\varepsilon .
$$

Therefore, $\phi_{\varepsilon}\left(z_{i}\right) \in[\varepsilon, 1-\varepsilon] \subset\left[a_{i}, b_{i}\right)$. Assume $i \in A^{\sigma}$. Note that in this case

$$
z_{i} \in\left[x_{i}, y_{i}\right) \subset\left[a_{i}, b_{i}\right),
$$

and $b_{i}-a_{i} \geq \varepsilon$ (otherwise $|B|<\varepsilon$ ). If $\varepsilon \leq z_{i} \leq 1-\varepsilon$ then $\phi_{\varepsilon}\left(z_{i}\right)=z_{i}$ hence $\phi_{\varepsilon}\left(z_{i}\right) \in\left[a_{i}, b_{i}\right)$. If $0 \leq z_{i}<\varepsilon$ then the interval $\left[a_{i}, b_{i}\right)$ contains a point smaller than $\varepsilon$ and has length at least $\varepsilon$. Then it must contain $\varepsilon=\phi_{\varepsilon}\left(z_{i}\right)$. Similarly, if $1-\varepsilon<z_{i}<1$ then $\left[a_{i}, b_{i}\right)$ must contain $1-\varepsilon=\phi_{\varepsilon}\left(z_{i}\right)$. This proves that if $z \in B_{0}$ then $\phi_{\varepsilon}(z) \in B$. Thus, $\mathcal{N}$ is a $(1 / 2)$-dinet for $\mathcal{B}_{\varepsilon, d}$. This completes the proof.

\section{Acknowledgments}

The author was introduced to this problem during the 2017 MFO workshop "Perspectives in High-dimensional Probability and Convexity." The author is grateful to MFO, to the organizers, and participants of the workshop. The author is also grateful to A. Zelnikov for his help with the picture.

\section{References}

[1] C. Aistleitner, A. Hinrichs, D. Rudolf, On the size of the largest empty box amidst a point set, Discrete Appl. Math. 230 (2017), 146-150.

[2] A. Blumer, A. Ehrenfeucht, D. Haussler, M. Warmuth, Learnability and the Vapnik-Chervonenkis dimension, J. Assoc. Comput. Mach. 36 (1989), 929-965.

[3] A. Dumitrescu, M. Jiang, On the largest empty axis-parallel box amidst $n$ points, Algorithmica 66 (2013), 225-248. 
[4] M. Gnewuch, Bracketing numbers for axis-parallel boxes and applications to geometric discrepancy, J. Complexity 24 (2008), 154-172.

[5] A. Hinrichs, D. Krieg, R.J. Kunsch, D. Rudolf, Expected dispersion of uniformly distributed points, J. Complexity, to appear.

[6] A. Hinrichs, J. Prochno, M. Ullrich, J. Vybíral, The minimal $k$ dispersion of point sets in high-dimensions, J. Complexity, 51 (2019), 68-78.

[7] E. Hlawka, Abschätzung von trigonometrischen Summen mittels diophantischer Approximationen, Österreich. Akad. Wiss. Math.Naturwiss. Kl. S.-B. II, 185 (1976), 43-50.

[8] D. Krieg, On the dispersion of sparse grids, J. Complexity 45 (2018), $115-119$.

[9] G. Rote, R.F. Tichy, Quasi-Monte Carlo methods and the dispersion of point sequences, Math. Comput. Modelling 23 (1996), 9-23.

[10] D. Rudolf, An upper bound of the minimal dispersion via delta covers, Contemporary Computational Mathematics - A Celebration of the 80th Birthday of Ian Sloan, Springer-Verlag, (2018), 1099-1108.

[11] J. Sosnovec, A note on the minimal dispersion of point sets in the unit cube, European J. of Comb., 69 (2018), 255-259.

[12] V.N. Temlyakov, Dispersion of the Fibonacci and the Frolov point sets, preprint, 2017, arXiv:1709.08158.

[13] M. Ullrich, A lower bound for the dispersion on the torus, Mathematics and Computers in Simulation 143 (2018), 186-190.

[14] M. Ullrich, A note on the dispersion of admissible lattices, Discrete Appl. Math., 257 (2019), 385-387.

[15] M. Ullrich, J. Vybíral, An upper bound on the minimal dispersion, Journal of Complexity 45 (2018), 120-126.

[16] M. Ullrich, J. Vybíral, Deterministic constructions of high-dimensional sets with small dispersion, Preprint, 2019, arXiv:1901.06702 


\begin{abstract}
Alexander Litvak
Dept. of Math. and Stat. Sciences, University of Alberta,

Edmonton, AB, Canada, T6G 2 G1.

e-mail: alitvak@ualberta.ca
\end{abstract}

